\title{
THE DERIVATIVE OF THE EXPONENTIAL MAP
}

\author{
CARL HERZ
}

(Communicated by Jonathan M. Rosenberg)

\begin{abstract}
We give a quick analytic derivation of the formula for the derivative of the exponential of a vector field on a manifold.
\end{abstract}

Our object is to give a quick proof of a result usually obtained only for analytic manifolds using combinatorial manipulation with power series.

Let $\mathscr{M}$ be a $\mathrm{C}^{(\infty)}$-manifold. Put $\operatorname{VEC}(\mathscr{M})$ for the space of $\mathrm{C}^{(\infty)}$-vector fields on $\mathscr{M}$. Given $X \in \operatorname{VeC}(\mathscr{M})$ and $p \in \mathscr{M}$ we write $\exp (t X) p$ for the point on the manifold corresponding to the flow of $X$ at time $t$ that passes through $p$ at time $t=0$. Thus one has

$$
\left.(d / d t) f \circ \exp (t X)\right|_{t=0}=f^{\prime} \circ X
$$

everywhere on $\mathscr{M}$, where $f^{\prime}$ is the derivative of the mapping $f: \mathscr{M} \rightarrow \mathbb{R}$. If $\exp (t X)$ is globally defined then

$$
(d / d t) f \circ \exp (t X)=f^{\prime} \circ X \circ \exp (t X)=f^{\prime} \circ T(\exp (t X)) \circ X .
$$

Here we use the notation $T(S): T(\mathscr{M}) \rightarrow T(\mathscr{M})$ for the functorial map of the tangent bundle corresponding to a $\mathrm{C}^{(\infty)}$-map $S: \mathscr{M} \rightarrow \mathscr{M}$.

Let $S$ be an automorphism of $\mathscr{M}$. Given $V \in \operatorname{VEC}(\mathscr{M})$ we get a new vector field

$$
(\operatorname{Ad} S) V \stackrel{\text { def }}{=} T(S) \circ V \circ S^{-1} \text {. }
$$

(1) Proposition. Suppose $X, V \in \operatorname{Vec}(\mathscr{M})$ and that $\exp X$ is globally defined. Then, for any test function $f \in \mathrm{C}^{(\infty)}(\mathscr{M})$ we have

$$
\left.(d / d t) f \circ(\exp (X+t V)) \circ \exp (-X)\right|_{t=0}=f^{\prime} \circ \int_{0}^{1} \operatorname{Ad}(\exp s X) V d s .
$$

Remark. $X$ and $V$ are fixed in the statement. Thus, for each $p \in \mathscr{M}$, $s \mapsto \operatorname{Ad}(\exp s X) V(p)$ gives a continuous map $[0,1] \rightarrow T_{p}(\mathscr{M}) . f^{\prime}$ is a linear functional on the finite-dimensional vector space $T_{p}(\mathscr{M})$. Thus one has an

Received by the editors June 6, 1990 and, in revised form, November 21, 1990.

1991 Mathematics Subject Classification. Primary 58A30, 22E30.

Key words and phrases. Vector field, exponential map.

Research supported by the Natural Science and Engineering Research Council of Canada and les Fonds FCAC du Québec. 
ordinary vector-valued integral that commutes with the action of linear functionals.

Proof. For $0 \leq s \leq 1$ and small $t$ put

$$
F(s, t)=f \circ \exp (s(X+t V)) \circ \exp (-s X) .
$$

It is clear that one gets a vector field $W_{s}$ defined, for $s>0$, by

$$
f^{\prime} \circ s W_{s}=\left.(\partial / \partial t) F(s, t)\right|_{t=0} .
$$

On the other hand we have

$$
(\partial / \partial s) F(s, t)=f^{\prime} \circ T(\exp (s(X+t V))) \circ t V \circ \exp (-s X) .
$$

If we differentiate the line above with respect to $t$ at $t=0$, then, using the chain rule, all terms drop out except the differentiation of $t V$, and we get

$$
f^{\prime} \circ \operatorname{Ad}(\exp s X) V \text {. }
$$

Comparing the derivative of (2) with respect to $s$ and the derivative of (3) with respect to $t$ at $t=0$, one has

$$
(d / d s) s f^{\prime} \circ W_{s}=f^{\prime} \circ \operatorname{Ad}(\exp s X) V,
$$

and integrating from 0 to 1 gives the conclusion.

Remark. It is not necessary that $\exp X$ be globally defined. The formula of Proposition (1) holds at any point $p \in \mathscr{M}$ such that the flow of $X$ with initial condition at $p$ is defined in a neighborhood of the time interval $[-1,0]$; this is to say that $\exp (-X) p$ is defined.

The commutator of vector fields defined on test functions by

$$
f^{\prime} \circ[V, W]=\left(f^{\prime} \circ V\right)^{\prime} \circ W-\left(f^{\prime} \circ W\right)^{\prime} \circ V .
$$

A straightforward calculation gives

$$
(d / d s) \operatorname{Ad}(\exp s X) V=\operatorname{Ad}(\exp s X)[X, V]=[X, \operatorname{Ad}(\exp s X) V] .
$$

Thus ad $X$ defined on $\operatorname{VeC}(\mathscr{M})$ by ad $X V=[X, V]$ is the infinitesimal generator of the one-parameter group of operators $s \mapsto \operatorname{Ad}(\exp s X)$. We may therefore write

$$
\operatorname{Ad}(\exp s X) \equiv \exp (s \operatorname{ad} X)
$$

with the understanding that the right-hand side is to be interpreted in the general case as the left-hand side. On an analytic manifold one may expand

$$
\exp (s \operatorname{ad} X) V=\sum_{k=0}^{\infty}(1 / k !) s^{k}(\operatorname{ad} X)^{k} V .
$$

This formula remains valid if there exists a finite-dimensional Lie algebra $\mathfrak{g} \subset$ $\operatorname{VeC}(\mathscr{M})$ such that $X, V \in \mathfrak{g}$. Let us define

$$
\psi(u)=\int_{0}^{1} e^{s u} d s=u^{-1}\left(e^{u}-1\right) .
$$


The formula of (1) may be rewritten to give

(6) Theorem. If $X, V \in \operatorname{Vec}(\mathscr{M})$ then at any point $p \in \mathscr{M}$ for which $\exp (-X) p$ is defined we have (composition with test functions being understood)

$$
\left.(d / d t) \exp (X+t V) \circ \exp (-X)\right|_{t=0}=\psi(\operatorname{ad} X) V .
$$

This formula with $\psi$ expanded as a power series gives the usual expression, [1, Chapter II, Theorem 1.7]. (The difference in sign results from the fact that we have differentiation acting on the right instead of the left.) In the context of Lie groups, there is a somewhat longer proof in the same spirit as ours in [2, Theorem 2.14.2]. Note that the power series expansion is valid not only on analytic manifolds but also if $\mathscr{M}$ is merely a $\mathrm{C}^{(2)}$-manifold provided that $X$ and $V$ are $\mathrm{C}^{(2)}$-vector fields generating a finite-dimensional Lie algebra.

In the application to Lie groups $\mathbf{G}$ one considers the left multiplication operator $L_{S}$ and the right multiplication operator $R_{S}$ corresponding to an element $S \in \mathbf{G}$. The Lie algebra $\mathfrak{g}$ is identified as $T_{I}(\mathbf{G})$, and one associates to $X \in \mathfrak{g}$ the infinitesimal left translation (right-invariant vector field) $\Lambda_{X}$ by defining $\Lambda_{X}(S)=T\left(R_{S}\right) X$. The bracket in the Lie algebra is given by $[X, Y]=\left[\Lambda_{X}, \Lambda_{Y}\right](I)$. Since left- and right-multiplication commute, we have $\left(\operatorname{Ad} L_{S}\right) \Lambda_{X}=\Lambda_{Y}$ where $Y=(\operatorname{Ad} S) X$ in the usual sense of the adjoint action of $\mathbf{G}$ on $\mathfrak{g}$. Thus (7) extends the standard formula for Lie groups to general vector fields on a manifold.

\section{REFERENCES}

1. S. Helgason, Differential geometry, Lie groups, and symmetric spaces, Academic Press, New York, 1978.

2. V.S. Varadarajan, Lie groups, Lie algebras, and their representations, Prentice-Hall, Englewood Cliffs, NJ, 1974.

Department of Mathematics and Statistics, McGill University, 805 Sherbrooke Street West, Montreal H3A 2K6, Canada 